Supporting Information

\title{
Encapsulation of a Monolayer WSe 2 Phototransistor with Hydrothermally Grown ZnO Nanorods
}

Kang-Nyeoung Lee ${ }^{\dagger}, \S, \#$, Seungho Bang ${ }^{\dagger,+}, \#$, Ngoc Thanh Duong ${ }^{\dagger}$, Seok Joon Yun ${ }^{\dagger}+\dot{*}$, Dae Young Park ${ }^{\dagger}$, , Juchan Lee ${ }^{\dagger}$, Young Chul Choi $*, \xi$ and Mun Seok Jeong $*,+\neq$

${ }^{\dagger}$ Department of Energy Science, Sungkyunkwan University, Suwon 16419, Republic of Korea

* Center for Integrated Nanostructure Physics, Institute for Basic Science, Suwon 16419,

Republic of Korea

${ }^{\S}$ Korea Institute of Carbon Convergence Technology, Jeonju 54853, Republic of Korea

* Address correspondence to mjeong@skku.edu; youngchoi@ kctech.re.kr.

KEYWORDS: encapsulation, tungsten diselenide, zinc oxide, p-doping, antenna effect, charge transfer 


\section{Supplementary Figures}

S1. AFM image of ${ }^{1 L} \mathrm{WSe}_{2}$

S2. SEM images of ZnO NRs by $\mathrm{pH} .7$ conditions at various temperatures

S3. SEM images of ZnO NRs by pH.9 conditions at various temperatures

S4. SEM images of ZnO NRs by pH.11 conditions at various temperatures

S5. PL, XRD, and Raman to determine the quality of ZnO NRs

S6. Integrated PL intensity mapping images of etched pristine ${ }^{1 L} \mathrm{WSe}_{2}$ and $\mathrm{HS}_{-}{ }^{1 L} \mathrm{WSe}_{2}$ without degraded region

S7. Detailed process sequence for HS-phototransistor

S8. Photoinduced source-drain I-V curves $(-1$ to $+1 \mathrm{~V})$ with $V_{b g}-30 \mathrm{~V}$

S9. PL spectra of I (core region), II (central region), and III (edge region)

S10. Increment in PL intensity with quantitative data

S11. Field-effect mobility versus $V_{g s}$ of pristine ${ }^{1 L} W_{S} e_{2}$ phototransistor and HSphototransistor

S12. Comparison of hysteresis properties between pristine ${ }^{1 L} \mathrm{WSe}_{2}$ phototransistor and HSphototransistor

S13. Change in field-effect mobility for 14 days

\section{Supplementary Table}

Table S1. Summary of growth conditions of ZnO NRs using the hydrothermal method 


\section{S1. AFM image of ${ }^{1 \mathrm{~L}} \mathrm{WSe}_{2}$}

Figures S1a and S1b show the optical microscope image of CVD-grown $\mathrm{WSe}_{2}$ and the AFM image of the dashed area in Figure S1a, respectively. In Figure S1b, the thickness of $\mathrm{WSe}_{2}$ is observed as $\sim 0.94 \mathrm{~nm}$. Therefore, we could confirm a monolayer $\mathrm{WSe}_{2}\left({ }^{1 \mathrm{~L}} \mathrm{WSe}_{2}\right)$.
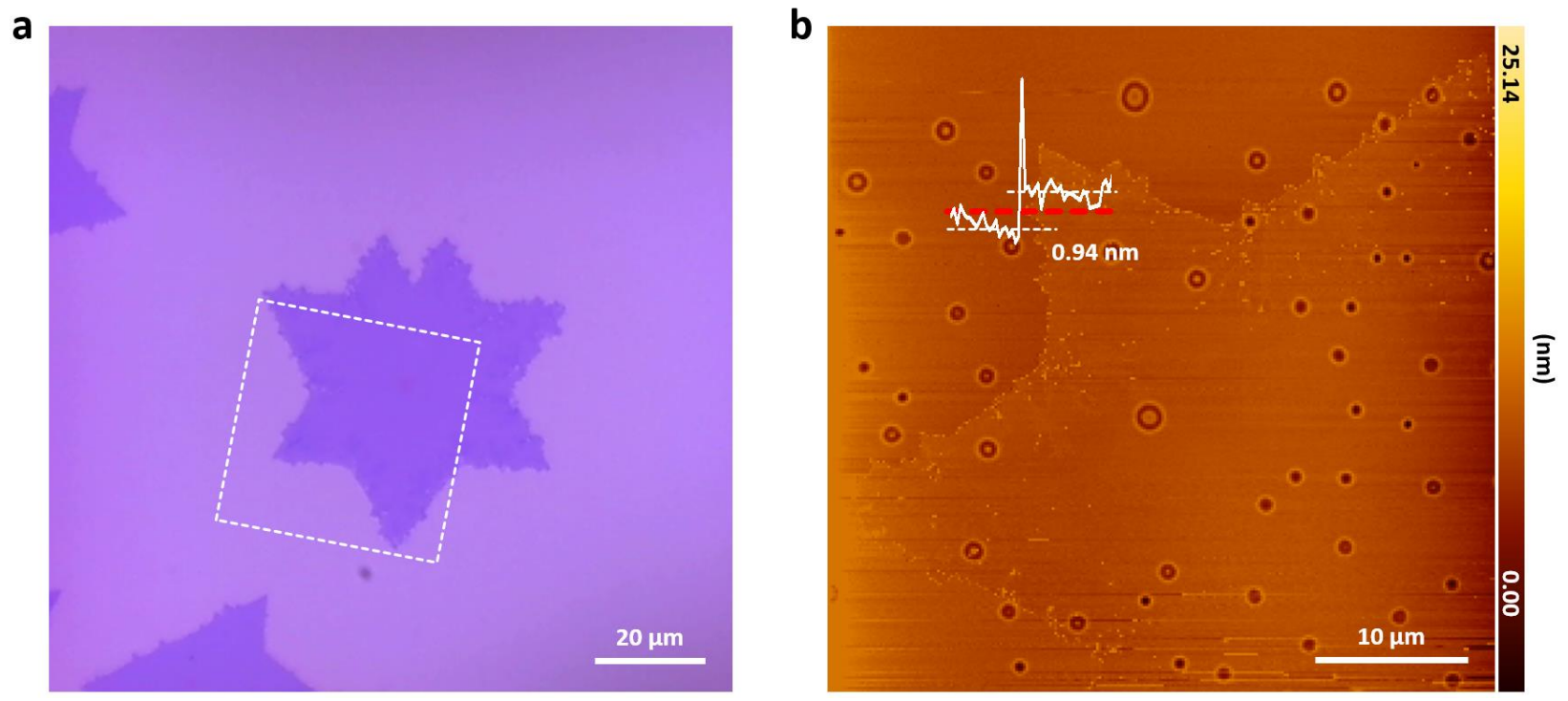

Figure S1. (a) Optical microscope image of transferred CVD-grown ${ }^{1 \mathrm{~L}} \mathrm{WSe}_{2}$. (b) AFM image of inside of the dashed line in Figure S1a. 


\section{S2. SEM images of $\mathrm{ZnO}$ NRs by pH.7 conditions at various temperatures}

Figure S2 shows the SEM images of hydrothermally grown ZnO NRs by pH.7 conditions at various temperatures $\left(130,150\right.$, and $\left.170{ }^{\circ} \mathrm{C}\right)$. We can confirm that the condition of pH.7, which exhibits a deficient growth rate, is not suitable for the synthesis of $\mathrm{ZnO}$ NRs.

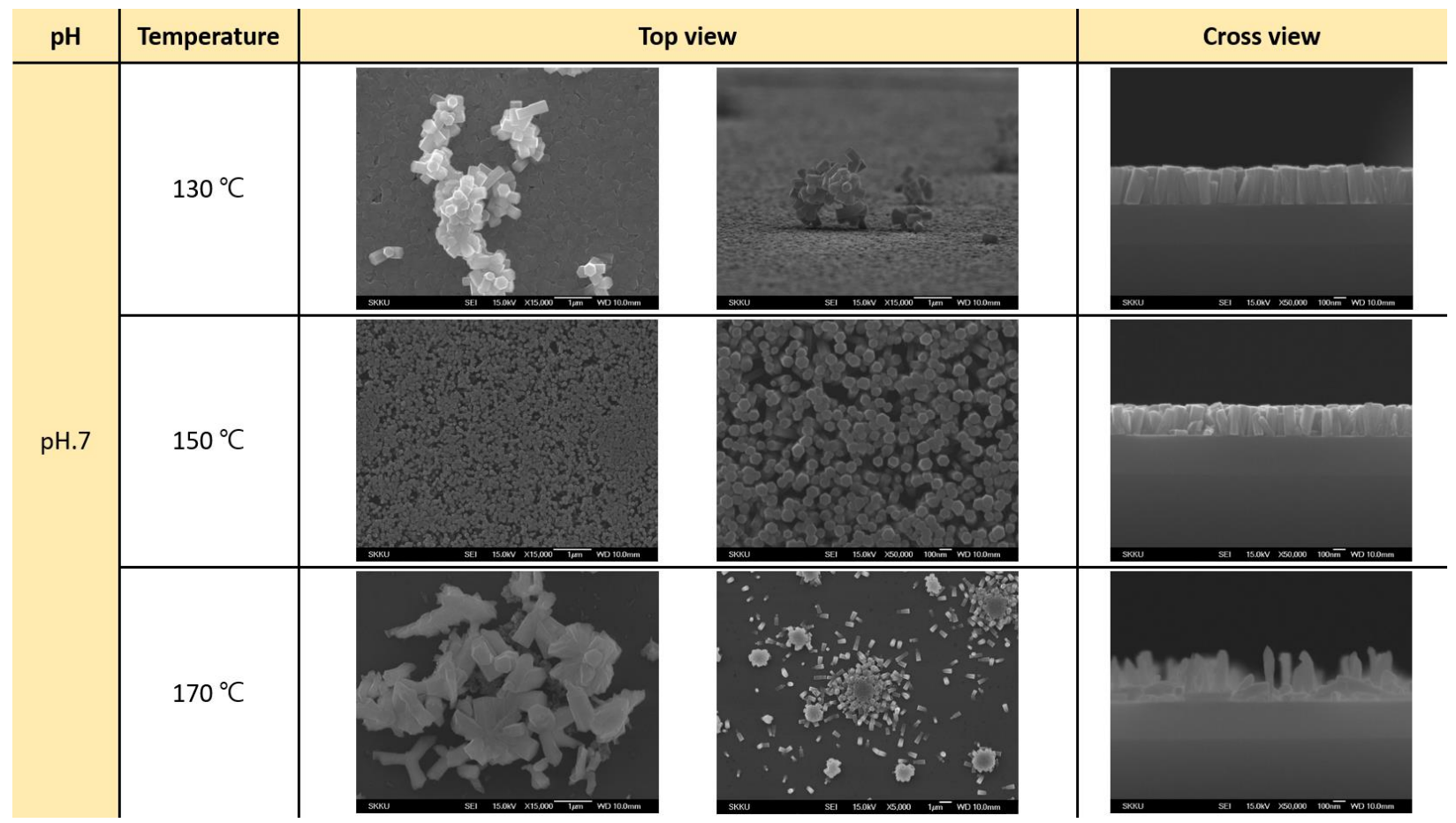

Figure S2. SEM images of ZnO NRs by pH.7 conditions at various temperatures $(130,150$, and $\left.170{ }^{\circ} \mathrm{C}\right)$. 


\section{S3. SEM images of $\mathrm{ZnO}$ NRs by pH.9 conditions at various temperatures}

Figure $\mathrm{S} 3$ shows the SEM images of hydrothermally grown ZnO NRs by pH.9 conditions at various temperatures $\left(130,150\right.$, and $\left.170{ }^{\circ} \mathrm{C}\right)$. We confirmed that, compared to those of the pH.7 conditions in Figure S2, the ZnO NRs were well-grown with a high aspect ratio.

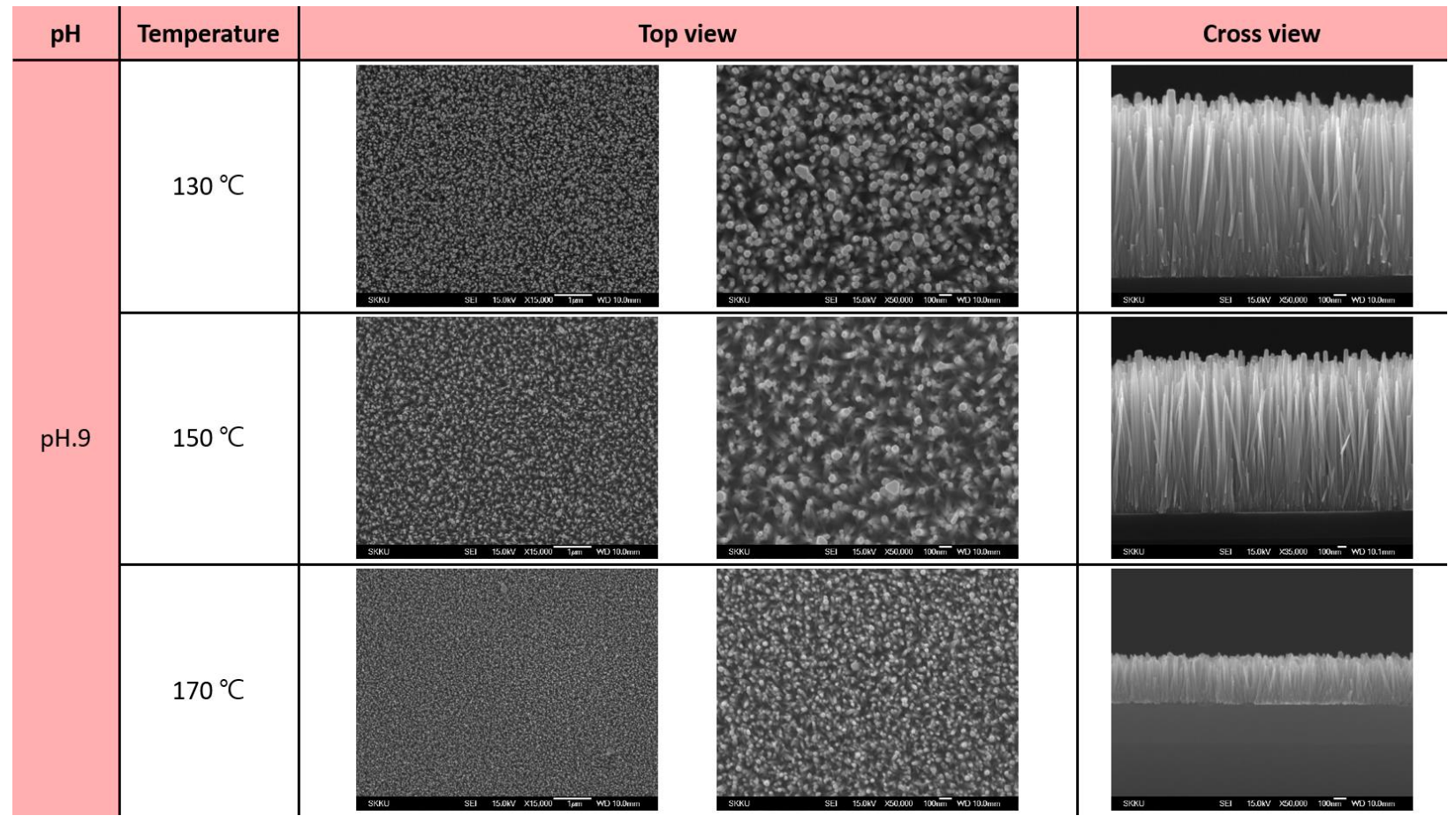

Figure S3. SEM images of $\mathrm{ZnO}$ NRs by pH.9 conditions at various temperatures $(130,150$, and $\left.170{ }^{\circ} \mathrm{C}\right)$. 


\section{S4. SEM images of ZnO NRs by pH.11 conditions at various temperatures}

Figure S4 shows the SEM images of hydrothermally grown $\mathrm{ZnO}$ NRs by $\mathrm{pH} .11$ conditions at various temperatures $\left(130,150\right.$, and $\left.170{ }^{\circ} \mathrm{C}\right)$. We confirmed that the condition of pH.11, similar to pH.9, is suitable for synthesizing $\mathrm{ZnO}$ NRs with a high aspect ratio.

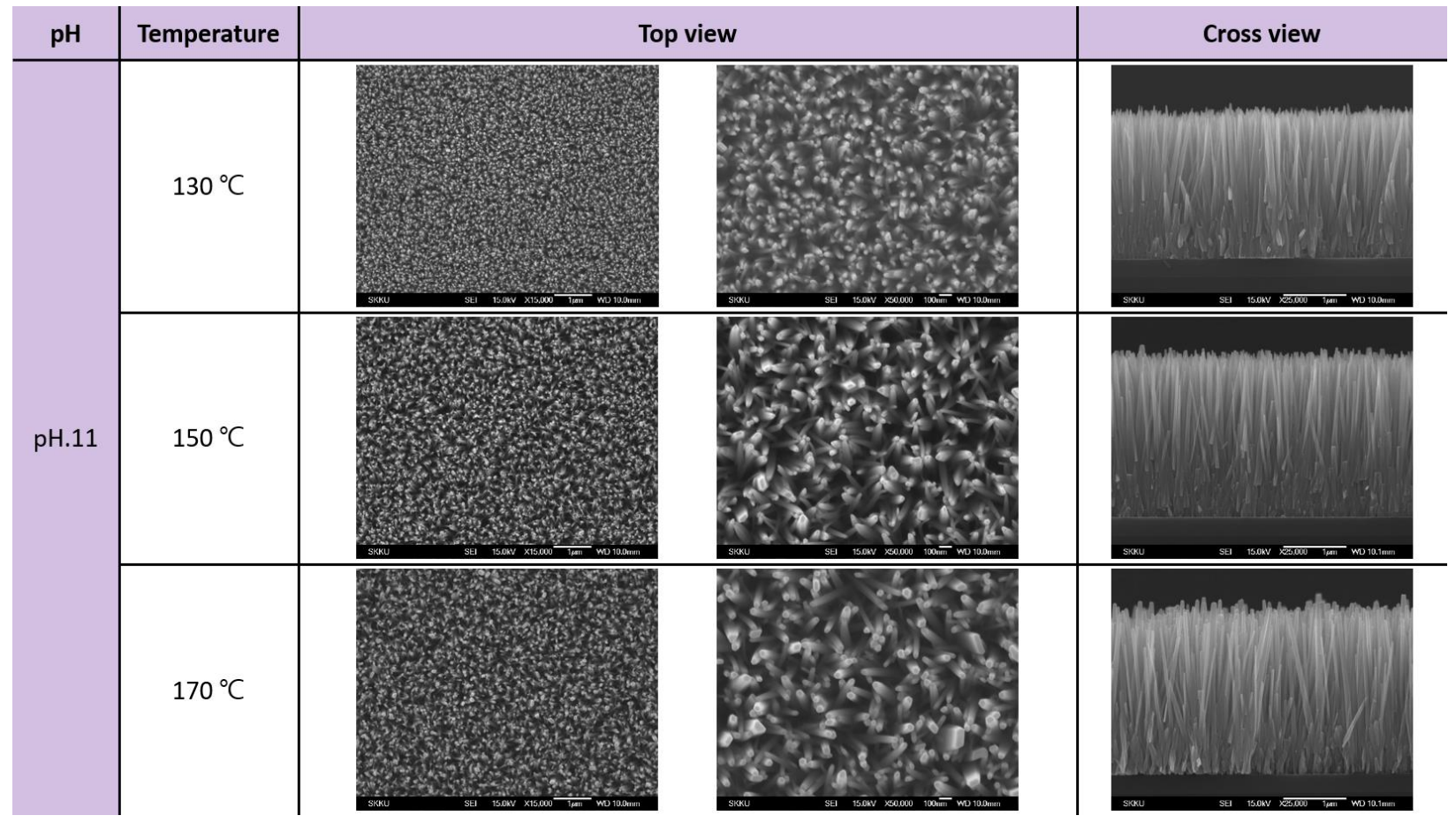

Figure S4. SEM images of ZnO NRs by pH.11 conditions at various temperatures $(130,150$, and $\left.170{ }^{\circ} \mathrm{C}\right)$. 


\section{S5. PL, XRD, and Raman to determine the quality of ZnO NRs}

Figures S5a-S5c, Figures S5d-S5f, and Figure S5g show the PL, XRD, and Raman of the ZnO NRs depending on various growth conditions using the hydrothermal method, respectively. For the PL and Raman measurements, $355 \mathrm{~nm}$ and $532 \mathrm{~nm}$ lasers were employed as an excitation source, respectively.

In Figure S5a, the ZnO NRs were synthesized by pH.7 conditions at $130{ }^{\circ} \mathrm{C}$ (green), $150{ }^{\circ} \mathrm{C}$ (yellow), and $170{ }^{\circ} \mathrm{C}$ (blue). We analyzed the intensity ratio of $\mathrm{NBE}$ versus $\mathrm{DL}\left(\mathrm{I}_{\mathrm{NBE}} / \mathrm{I}_{\mathrm{DL}}\right)$ to determine the quality of the $\mathrm{ZnO}$ NRs. Consequently, the $\mathrm{ZnO}$ NRs with the condition of "pH.7_130 ${ }^{\circ} \mathrm{C}^{\prime}$ indicated the highest ratio. In Figure S5b, the ZnO NRs were synthesized by pH.9 conditions at $130{ }^{\circ} \mathrm{C}$ (green), $150{ }^{\circ} \mathrm{C}$ (yellow), and $170{ }^{\circ} \mathrm{C}$ (blue). The $\mathrm{ZnO}$ NRs with the condition of "pH.9_150 ${ }^{\circ} \mathrm{C}$ " indicated the highest ratio. In Figure S5c, the ZnO NRs were synthesized by pH.11 conditions with $130{ }^{\circ} \mathrm{C}$ (green), $150{ }^{\circ} \mathrm{C}$ (yellow), and $170{ }^{\circ} \mathrm{C}$ (blue). The $\mathrm{ZnO}$ NRs with the condition of "pH.11_170 ${ }^{\circ} \mathrm{C}$ " indicated the highest ratio.

Figures S5d-S5f show the XRD patterns of the ZnO NRs with pH.7, pH.9, and pH.11 conditions, respectively. For each condition, the $\mathrm{ZnO}$ NRs were synthesized at various growth temperatures of $130{ }^{\circ} \mathrm{C}$ (green), $150{ }^{\circ} \mathrm{C}$ (yellow), and $170{ }^{\circ} \mathrm{C}$ (blue). Samples with many $\left(\begin{array}{lll}0 & 2\end{array}\right)$ planes in the $c$-axis direction were considered excellent. 
Figure S5g shows the Raman spectra based on each growth condition. We observed a relatively clear $\mathrm{E}_{2}$ mode in the $\mathrm{ZnO}$ NRs grown with $\mathrm{pH} .9$ and $\mathrm{pH} .11$ conditions.
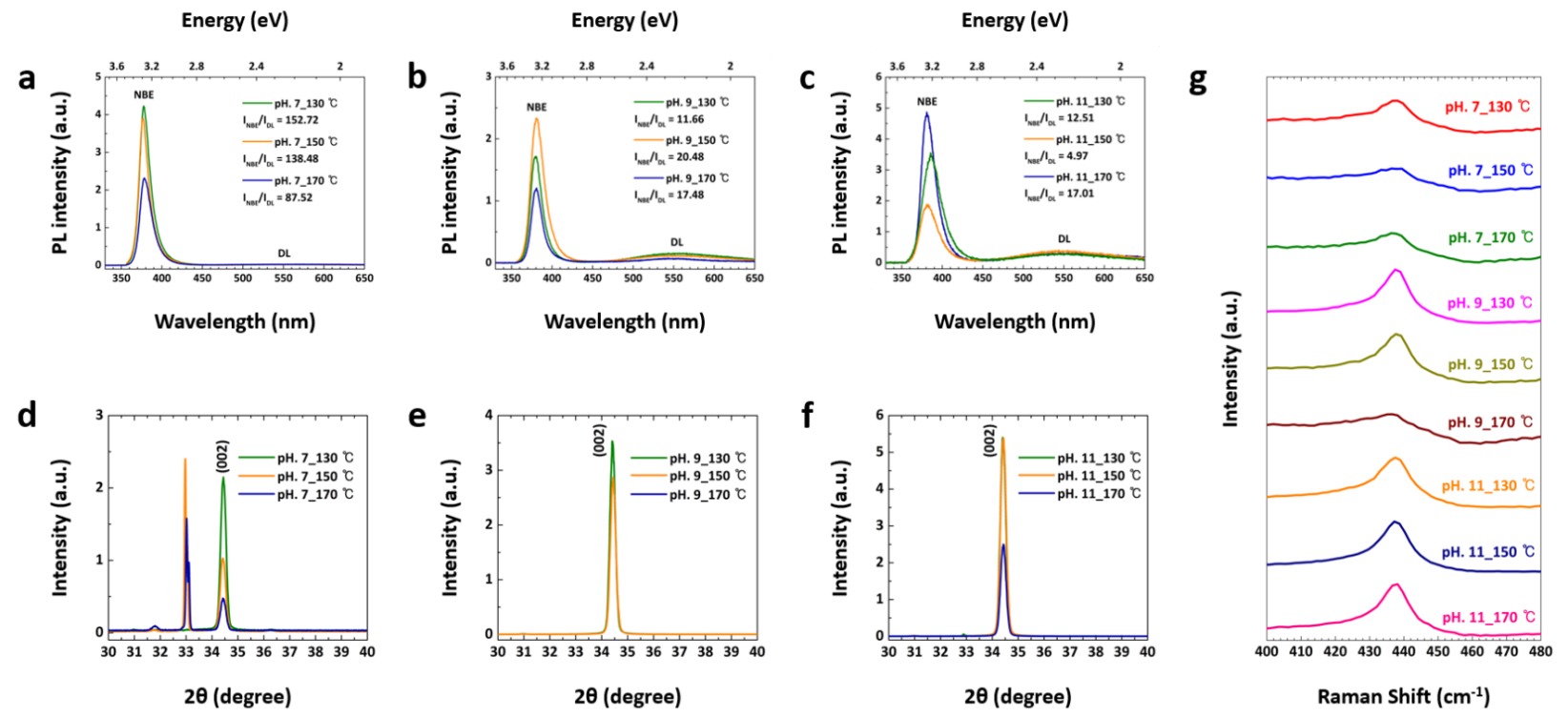

Figure S5. (a) PL spectra and (d) XRD patterns of ZnO NRs with pH.7 conditions. (b) PL spectra and (e) XRD patterns of ZnO NRs with pH.9 conditions. (c) PL spectra and (f) XRD patterns of ZnO NRs with pH.11 conditions. (g) Raman spectra of ZnO NRs at all conditions. 
S6. Integrated PL intensity mapping images of etched pristine ${ }^{1 \mathrm{~L}} \mathrm{WSe}_{2}$ and $\mathrm{HS}-{ }^{1 \mathrm{~L}} \mathrm{WSe}_{2}$ without degraded region

As shown in Figures $2 \mathrm{a}$ and $2 \mathrm{~b}$, the PL intensity was not enhanced at the edge regions (III). Through Raman spectroscopy, we confirmed a degraded pristine ${ }^{1 \mathrm{~L}} \mathrm{WSe}_{2}$ at the edge regions by $\mathrm{O}_{2}$ and $\mathrm{H}_{2} \mathrm{O}$ in the air, as shown in Figure 3a. To observe an entirely enhanced PL intensity of $\mathrm{HS}^{-1}{ }^{1 \mathrm{~L}} \mathrm{WSe}_{2}$ by the $\mathrm{ZnO} \mathrm{NRs}$, the pristine ${ }^{1 \mathrm{~L}} \mathrm{WSe}_{2}$ was etched to a rectangular shape by $\mathrm{SF}_{6}$ using the RIE system. After growing the $\mathrm{ZnO}$ NRs using the hydrothermal method, the PL intensity was enhanced significantly at the overall region of the etched $\mathrm{HS}-{ }^{1} \mathrm{WSe}_{2}$.
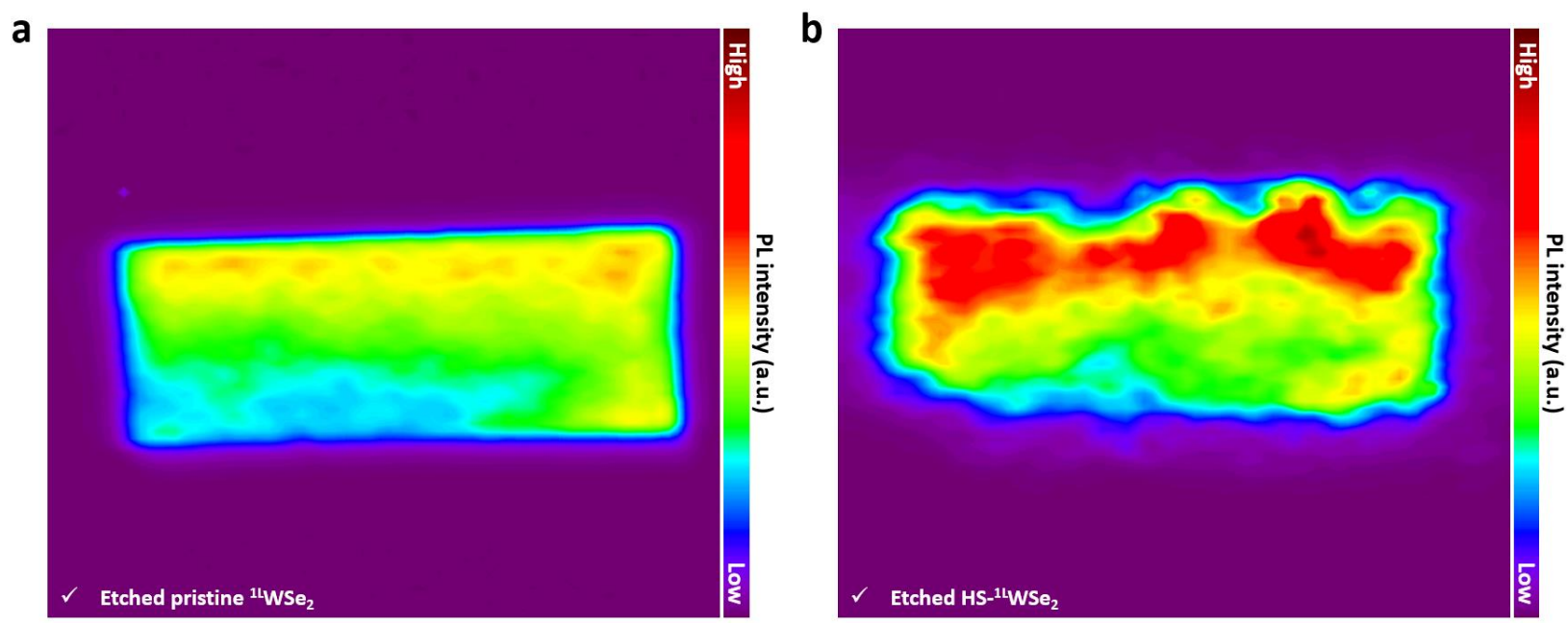

Figure S6. Integrated PL intensity mapping image of the (a) etched pristine ${ }^{1 \mathrm{~L}} \mathrm{WSe}_{2}$ and (b) etched $\mathrm{HS}-{ }^{1} \mathrm{LSe}_{2}$. 


\section{S7. Detailed process sequence for HS-phototransistor}

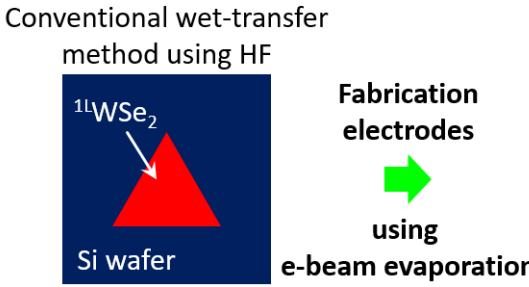

[Top view]

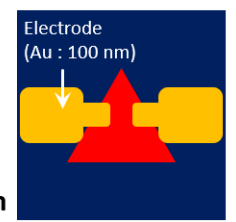

[Top view]

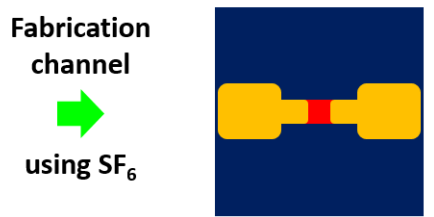

[Top view]

Electrical properties

of pristine ${ }^{1 \mathrm{~L}} \mathrm{WSe}_{2}$ phototransistor

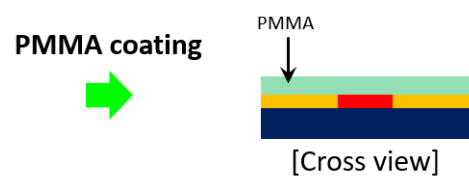

Fabrication open area [Cross view] e-beam lithography
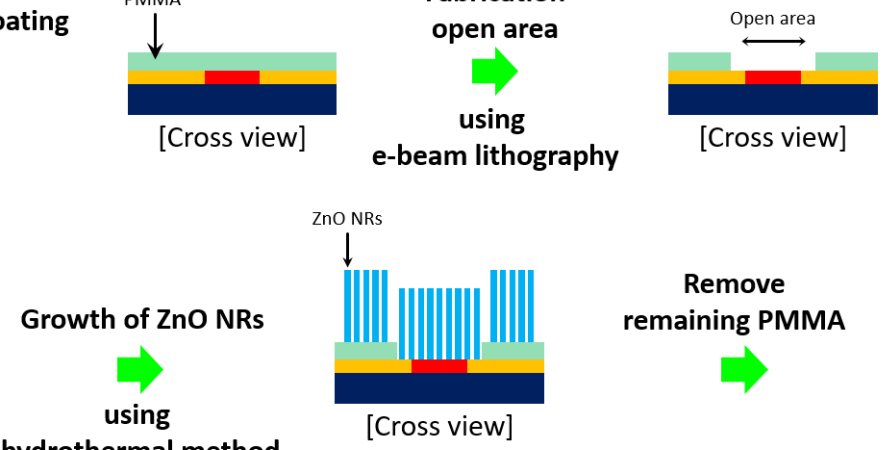

hydrothermal method

[Cross view]
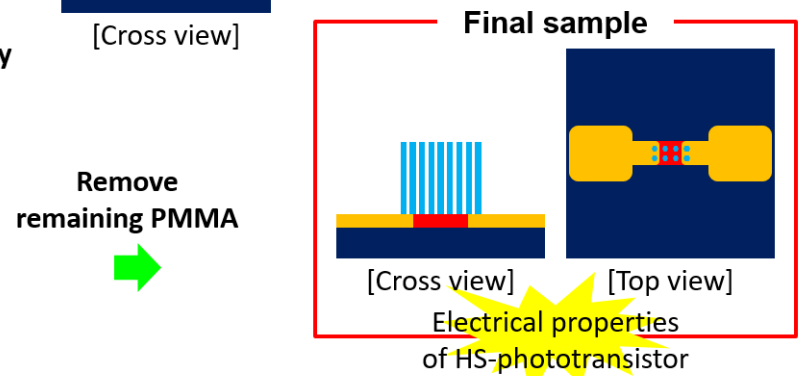

Figure S7. Detailed process sequence to fabricate HS-phototransistor. 


\section{S8. Photoinduced source-drain $I-V$ curves $(-1$ to $+1 \mathrm{~V})$ with $V_{b g}-30 \mathrm{~V}$}

Figures S8a and S8b show the S-D I-V curves (from -1 to $+1 \mathrm{~V}$ ) at a $-30 \mathrm{~V}$ back gate voltage in the pristine ${ }^{1} \mathrm{LSe}_{2}$ phototransistor and HS-phototransistor, respectively. A clearly enhanced photocurrent is observed after growing the $\mathrm{ZnO}$ NRs, as shown in Figure S8b. The photoresponsivity and photodetectivity of the HS-phototransistors were calculated to be $\sim 0.541$ $\mathrm{A} / \mathrm{W}$ and $4.23 \times 10^{9}$ Jones, respectively.

[Pristine ${ }^{1 \mathrm{~L}} \mathrm{WS} \mathrm{e}_{2}$ phototransistor]

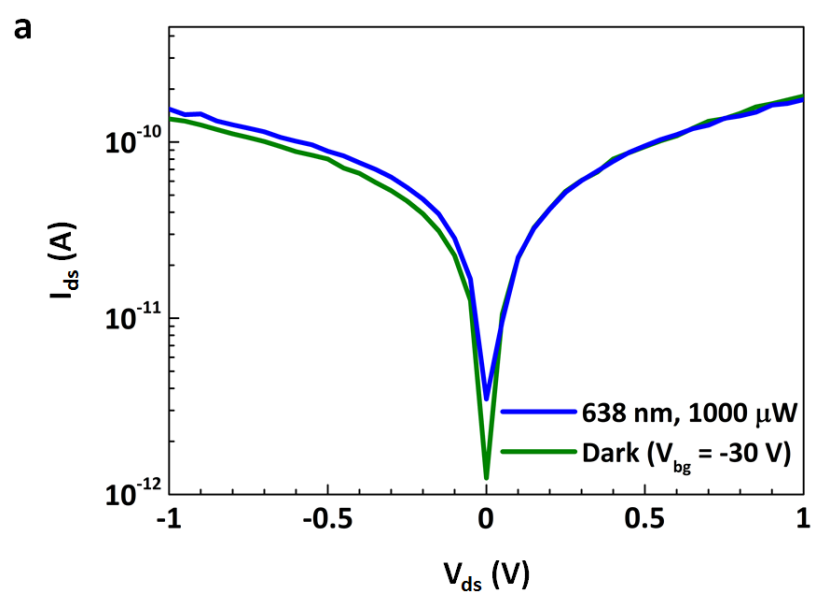

[HS-phototransistor]

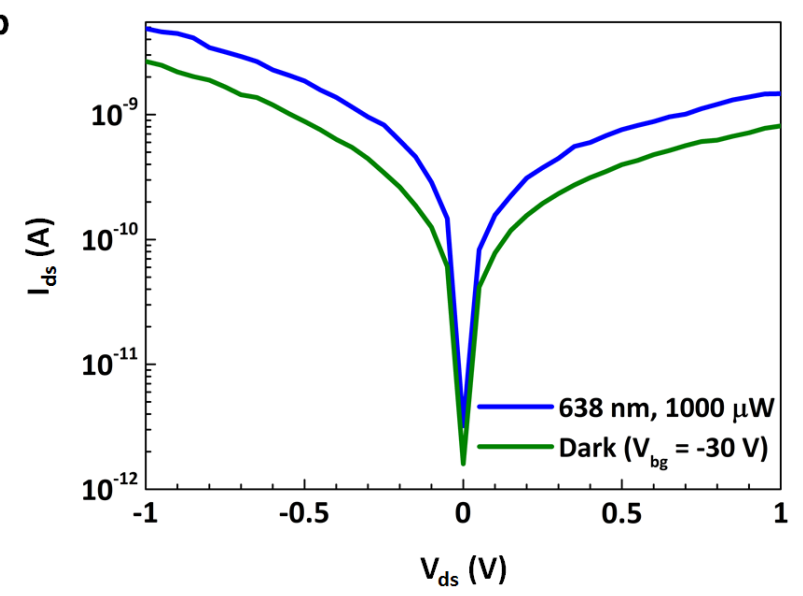

Figure S8. Photoinduced source-drain I-V curves ( -1 to $+1 \mathrm{~V})$ with $\mathrm{V}_{\mathrm{bg}}-30 \mathrm{~V}$ in the (a) pristine

${ }^{1 \mathrm{~L}} \mathrm{WSe}_{2}$ phototransistor and (b) HS-phototransistor. 


\section{S9. PL spectra of I (core region), II (central region), and III (edge region)}

Figures S9a-S9c show the PL spectra of regions I, II, and III in Figures 2a (black) and 2b (red), respectively. As shown in Figure S9a, the PL of pristine ${ }^{1 \mathrm{~L}} \mathrm{WSe}_{2}$ and $\mathrm{HS}^{-}{ }^{1 \mathrm{~L}} \mathrm{WSe}_{2}$ could not be observed owing to the lack of the as-grown ${ }^{1}{ }^{1} \mathrm{WSe}_{2}$ flake. In Figure S9b, notably, the enhanced PL of $\mathrm{HS}_{-}{ }^{1 \mathrm{~L}} \mathrm{WSe}_{2}$ was detected compared to that of pristine ${ }^{1 \mathrm{~L}} \mathrm{WSe}_{2}$ because of the charge transfer mechanism, as shown in Figure 2f, and the antenna effect. Figure S9c shows the same PL of pristine ${ }^{1} \mathrm{WSe}_{2}$ and $\mathrm{HS}-{ }^{1}{ }^{1} \mathrm{WSe}_{2}$ due to the degraded edge region, as mentioned in Figure 3a.
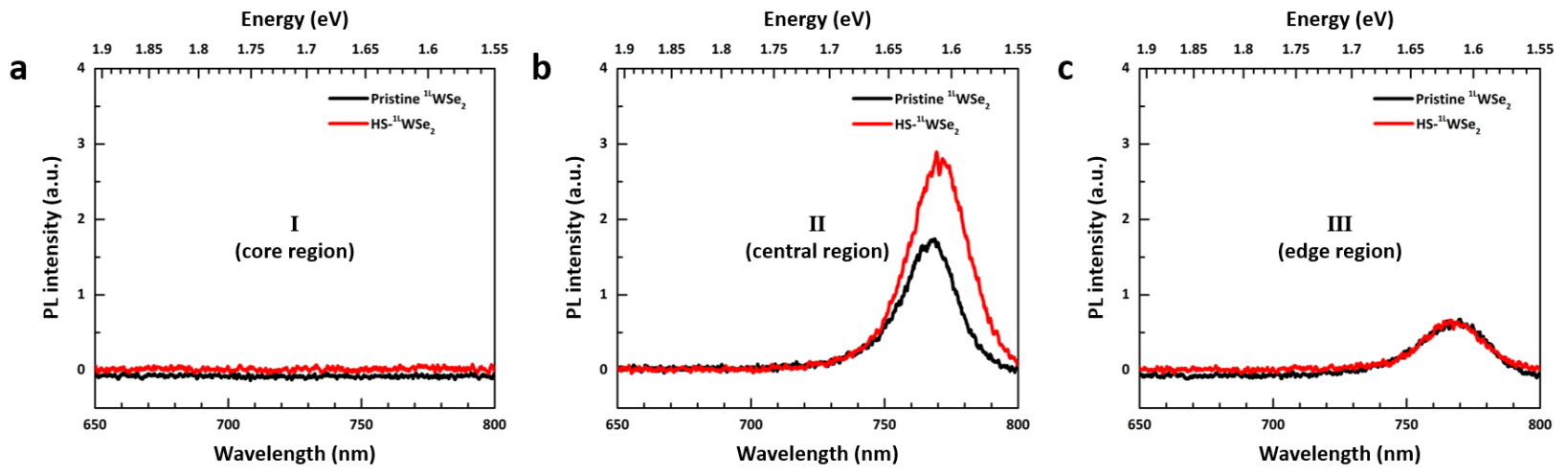

Figure S9. (a) PL spectra of region I (core region) in Figure 2a (black) and Figure 2b (red). (b) PL spectra of region II (central region) in Figure 2a (black) and Figure 2b (red). PL peaks A of pristine ${ }^{1 \mathrm{~L}} \mathrm{WSe}_{2}$ and $\mathrm{HS}^{1}{ }^{1 \mathrm{~L}} \mathrm{WSe}_{2}$ are located at $\sim 1.615 \mathrm{eV}$ and $\sim 1.610 \mathrm{eV}$, respectively. (c) PL spectra of region III (edge region) in Figure 2a (black) and Figure 2b (red). PL peaks A of pristine ${ }^{1 \mathrm{~L}} \mathrm{WSe}_{2}$ and $\mathrm{HS}_{-}{ }^{1 \mathrm{~L}} \mathrm{WSe}_{2}$ are both located at $\sim 1.615 \mathrm{eV}$. 


\section{S10. Increment in PL intensity with quantitative data}

Figure S10 provides specific evidence regarding the increment in PL intensity with quantitative data based on the same experimental data in Figure 2d. From the simple formula $((2,329-1,290) \times 100 / 1,290)$, it is apparent that the PL intensity of $\mathrm{HS}^{-1}{ }^{1} \mathrm{WSe}_{2}$ is $\sim 80 \%$ higher than that of pristine ${ }^{1 \mathrm{~L}} \mathrm{WSe}_{2}$.

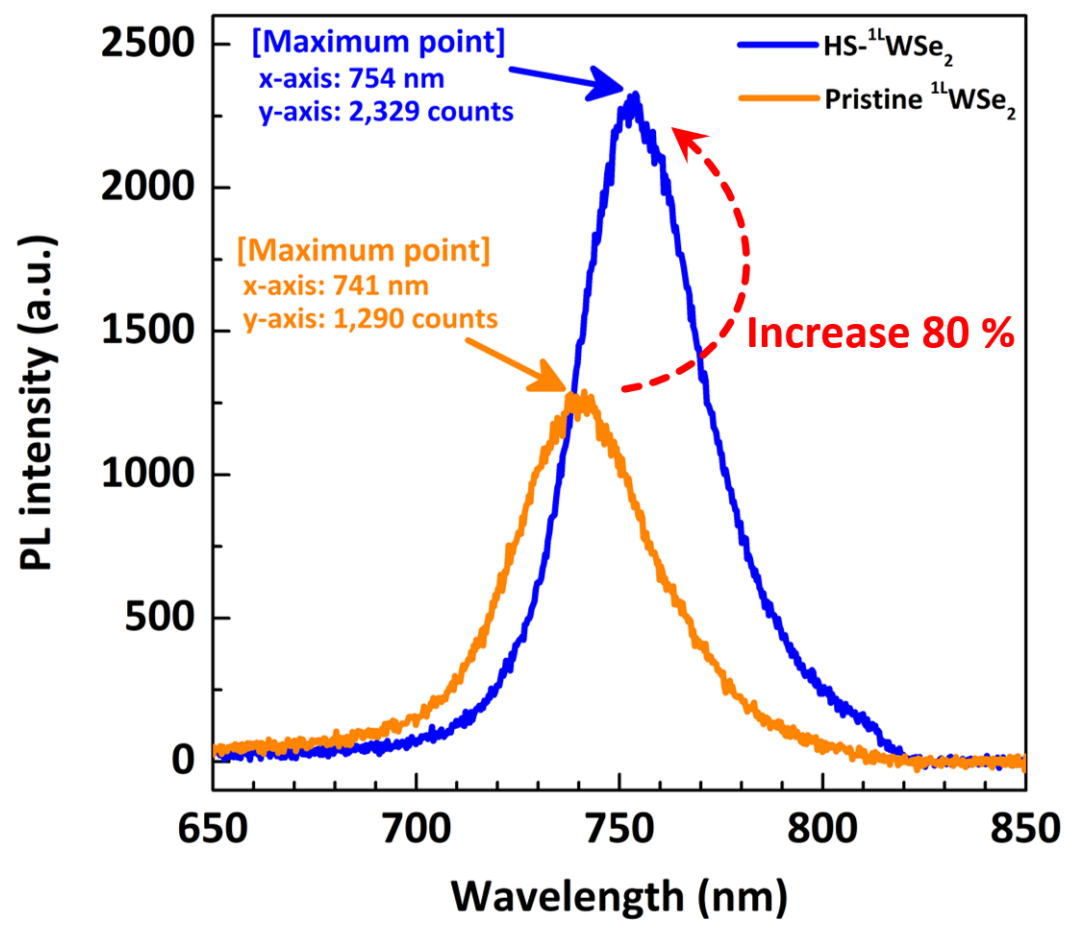

Figure S10. Maximum points of PL intensity for pristine ${ }^{1 \mathrm{~L}} \mathrm{WSe}_{2}$ (orange) and $\mathrm{HS}_{-}{ }^{1 \mathrm{~L}} \mathrm{WSe}_{2}$ (blue) are 1,290 (741 nm) and 2,329 (754 nm), respectively. 
S11. Field-effect mobility versus $\mathrm{V}_{\mathrm{gs}}$ of pristine ${ }^{1 \mathrm{~L}} \mathrm{WSe}_{2}$ phototransistor and HSphototransistor

The field-effect mobility of the HS-phototransistor is improved by a factor of 10 compared with the pristine ${ }^{1 \mathrm{~L}} \mathrm{WSe}_{2}$ photodetector owing to the p-doping effect. A positive shift in threshold voltage $\left(\mathrm{V}_{\text {th }}\right)$ is observed at the HS-phototransistor compared to the pristine ${ }^{1 \mathrm{~L}} \mathrm{WSe}_{2}$ phototransistor.

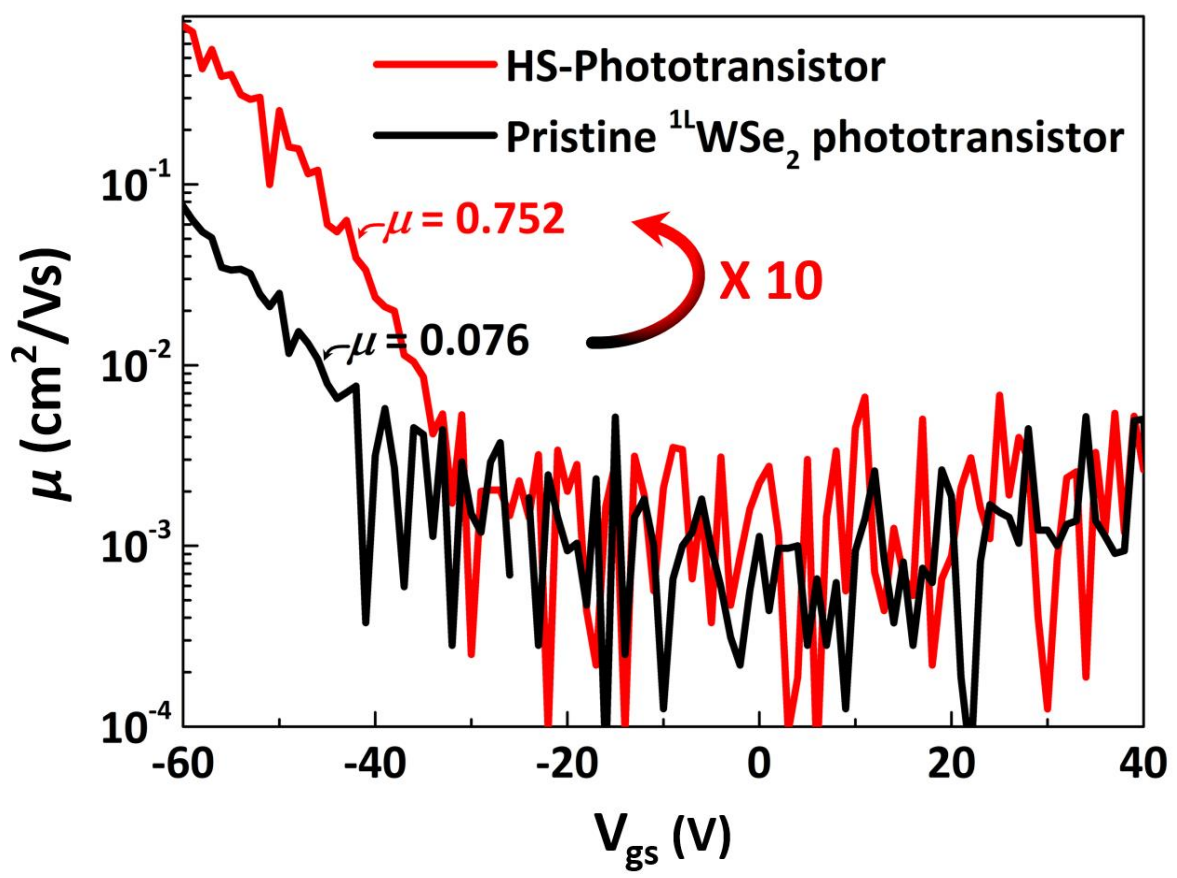

Figure S11. Transfer curves of field-effect mobility versus $\mathrm{V}_{\mathrm{gs}}$ of pristine ${ }^{1 \mathrm{~L}} \mathrm{WSe}_{2}$ phototransistor (black) and HS-phototransistor (red). 
S12. Comparison of hysteresis properties between pristine ${ }^{1 \mathrm{~L}} \mathrm{WSe}_{2}$ phototransistor and HSphototransistor

As shown in Figure $4 \mathrm{~b}$, we measured the gate-dependent $\mathrm{I}-\mathrm{V}$ curves of each phototransistor with the same gate sweep rate of $\sim 0.2 \mathrm{~V} / \mathrm{s}$. The hysteresis $\left(\Delta \mathrm{V}_{\text {th }}\right)$ is changed from 16 to $14 \mathrm{~V}$ after encapsulating the $\mathrm{ZnO}$ NRs.

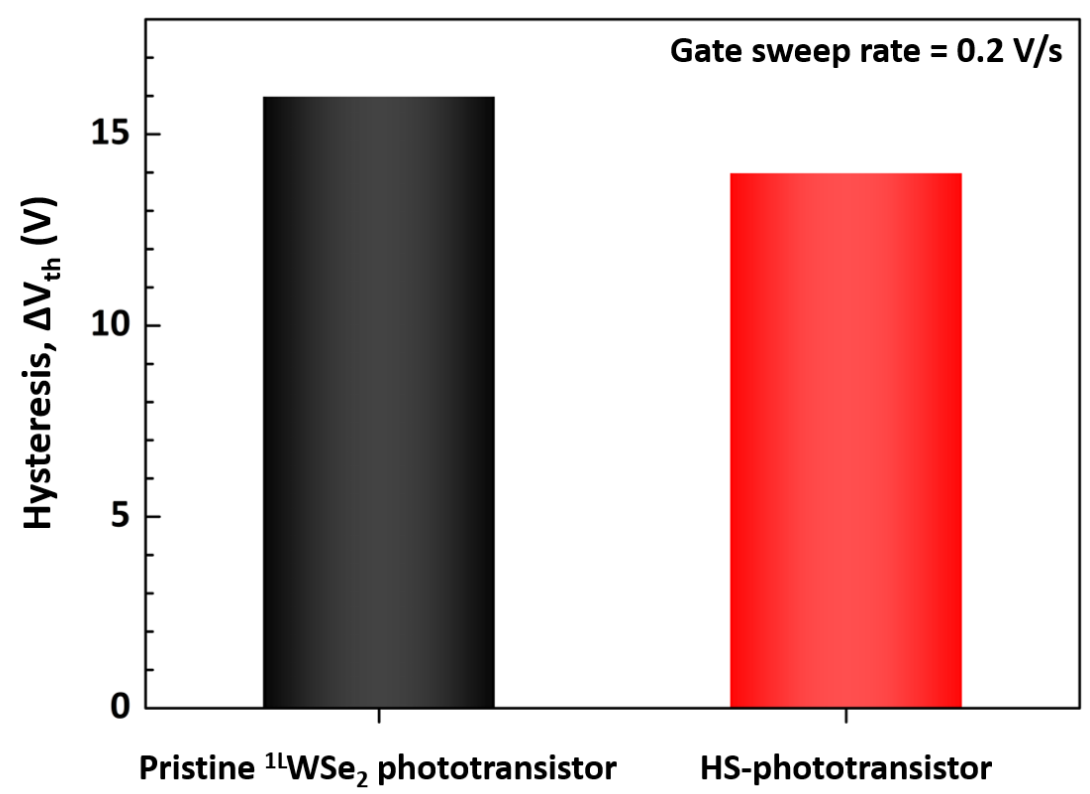

Figure S12. Hysteresis of pristine ${ }^{1} \mathrm{WSe}_{2}$ phototransistor and HS-phototransistor. 


\section{S13. Change in field-effect mobility for 14 days}

The hole mobility decreased from $0.75 \mathrm{~cm}^{2} / \mathrm{Vs}$ to $0.65 \mathrm{~cm}^{2} / \mathrm{Vs}$ after two weeks. The reduction rate of mobility is $13.3 \%$ calculated from $(0.75-0.65) / 0.75 \times 100$.

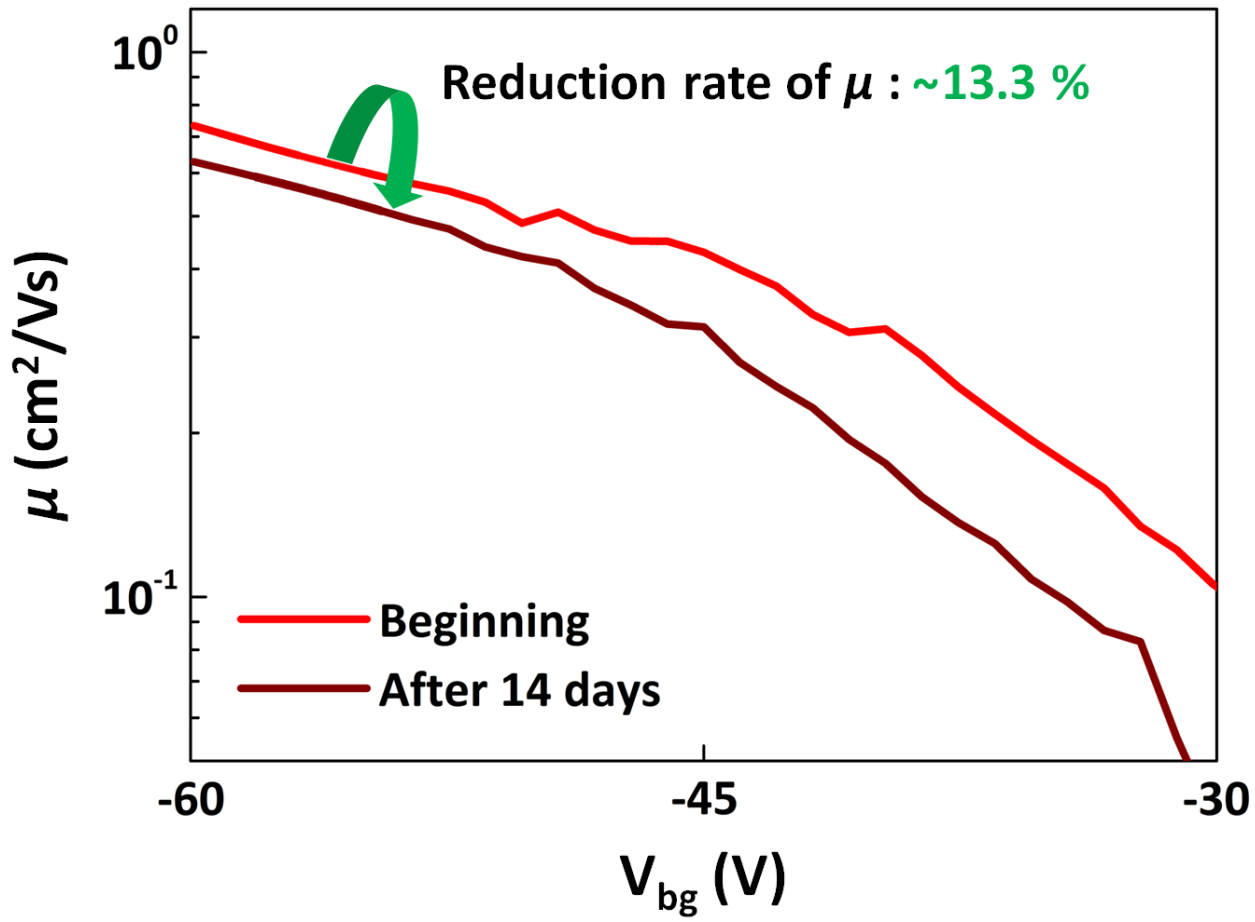

Figure S13. Field-effect mobility versus back gate voltage of the HS-phototransistor after two weeks. 
Table S1. Summary of growth conditions of ZnO NRs using the hydrothermal method

We present a table to confirm the high quality of the hydrothermally grown ZnO NRs. As shown in Table S1, we confirmed that the ZnO NRs of "pH.9_150 ${ }^{\circ} \mathrm{C}$ " exhibit fine optical and structural properties through Raman, PL, XRD, and SEM analysis.

$\begin{array}{cccc}\text { Condition for } & \text { Raman Shift } & \text { PL } & \text { XRD } \\ \text { ZnO nanorods } & {\left[\mathrm{E}_{2} \text { intensity }\right]} & {\left[\mathrm{I}_{\mathrm{NBE}} / \mathrm{I}_{\mathrm{DL}} \text { ratio }\right]} & {[(002) \text { peak intensity }]} \\ \text { growth } & & & \end{array}$

\begin{tabular}{|c|c|c|c|c|}
\hline pH.7_130 ${ }^{\circ} \mathrm{C}$ & Low & 152.72 & Low & $\mathrm{Bad}$ \\
\hline pH.7_150 ${ }^{\circ} \mathrm{C}$ & Low & 134.48 & None & $\mathrm{Bad}$ \\
\hline pH.7_170 ${ }^{\circ} \mathrm{C}$ & Low & 87.52 & None & $\mathrm{Bad}$ \\
\hline pH.9_130 ${ }^{\circ} \mathrm{C}$ & High & 11.66 & High & High \\
\hline pH.9_150 ${ }^{\circ} \mathrm{C}$ & High & 20.48 & High & High \\
\hline pH.9_170 ${ }^{\circ} \mathrm{C}$ & Low & 17.48 & Low & Low \\
\hline pH.11_130 ${ }^{\circ} \mathrm{C}$ & High & 12.51 & None & High \\
\hline pH.11_150 ${ }^{\circ} \mathrm{C}$ & High & 4.97 & High & High \\
\hline pH.11_170 ${ }^{\circ} \mathrm{C}$ & High & 17.01 & None & High \\
\hline
\end{tabular}

Table S1. Summary of growth conditions of $\mathrm{ZnO}$ NRs using the hydrothermal method. After confirming the conditions, we conclude that "pH.9_150 ${ }^{\circ} \mathrm{C}$ " is the best condition. 